\title{
Level set propagation for mixed-mode crack advance
}

\author{
D. Colombo ${ }^{\mathrm{a} *}$ and P. Massin ${ }^{\mathrm{b}}$ \\ ${ }^{a}$ School of Mechanical, Aerospace and Manufacturing Engineering, The University of Manchester, \\ Manchester, UK; ${ }^{b}$ LaMSID, UMR EDF-CNRS-CEA 2832, 1 Avenue du Général de Gaulle, 92141 \\ Clamart CEDEX, France
}

\begin{abstract}
In the extended finite element method framework, the advantage of being able to represent cracks independently from mesh rests on the use of level sets. Propagation simulation is connected to the update of these level sets, following the resolution of differential equations of evolution. In this document, we analyse these equations so that fast and robust mixed-mode propagation methods can be put forward. The proposed methodology can also manage the separation of a crack front into several fronts as well as the merging of several fronts during the propagation process. An example of 3D non-planar propagation is proposed at the end to show the efficiency of our choices.
\end{abstract}

Un des avantages de la méthode X-FEM est de permettre la représentation des fissures en utilisant le maillage sain d'une structure et leur localisation par des courbes de niveau. Leur propagation est alors réalisée par la mise à jour de ces courbes de niveau, dont l'évolution est obtenue par la résolution d'équations différentielles. Nous proposons d'analyser ici l'ensemble de ces équations pour aboutir à une méthode de propagation en mode mixte à la fois robuste et rapide. La méthodologie proposée permet en outre de pouvoir traiter la séparation du front de fissure en plusieurs fronts ainsi que la fusion de plusieurs fronts au cours de la propagation. Un exemple illustratif de propagation 3D hors plan est donné en fin d'article afin de démontrer l'efficacité des choix adoptés.

Keywords: X-FEM; level set; 3D propagation

Mots-clés: X-FEM; level set; propagation 3D

\section{Introduction}

Over the last decade, the extended finite element method (X-FEM) (Belytschko \& Black, 1999; Moës, Dolbow, \& Belytschko, 1999) coupled with a representation of cracks by level sets (Gravouil, Moës, \& Belytschko, 2002; Moës, Gravouil, \& Belytschko, 2002) has proven to be an interesting tool for crack propagation simulation, even if optimised meshing tools are still leading the way in terms of performance, such as ZENCRACK developed by Zentech or Z-set co-developed by the Ecole des Mines in Paris, MW Numerics and Onera, among others. The most interesting aspect of the X-FEM method is that cracks may be introduced into the structure, in which they may develop, independently of the initial mesh. In principle, therefore, the crack does not need to be meshed, or at least not in relation to the structural mesh. Discontinuity in the displacement field across the crack surface and the singularity of the stress field at the crack front are taken into account directly in the formulation of the finite

*Corresponding author. Email: daniele.colombo@manchester.ac.uk

ISSN 1779-7179 print/ISSN 1958-5829 online

(C) 2012 Taylor \& Francis

http://dx.doi.org/10.1080/17797179.2012.714849

http://www.tandfonline.com 
elements close to the crack (Moës et al., 1999)). The crack surface and front are located by the use of two families of level sets which are orthogonal to each other. The crack propagation may be taken into account by the change of these level sets, without the need to change the structural mesh (Gravouil et al., 2002).

Several techniques have been put forward to enhance the level set update: some of them concern the resolution of differential equations, which mathematically describe the problem of the level sets evolution (Adalsteinsson \& Sethian, 1995; Gravouil et al., 2002; Moës et al., 2002; Peng, Merriman, Osher, Zhao, \& Kang, 1999) while others use non-differential equations and geometric evolutions (Duflot, 2007; Ventura, Budyn, \& Belytschko, 2003). A comparison between these different methods can be found in Duflot (2007).

\section{Level sets propagation}

The level sets propagation stages are herein assessed so that $3 \mathrm{D}$ mixed-mode propagation and the consequent kink of the crack surface can be simulated. Whatever the method used, the propagation methodology described in Moës et al. (2002) can be adopted as a starting point on account of its wide applicability. The update process of level sets can be broken down into four stages. Firstly, level sets are updated in order to take into account crack propagation. Following this stage, the level sets no longer constitute distance functions and are no longer orthogonal to each other. Therefore a re-initialisation stage must be carried out for each level set, followed by a stage which makes the two level sets orthogonal to each other. A HamiltonJacobi (Moës et al., 2002) differential equation can be applied to each of these stages.

\subsection{Numerical resolution of the update stage}

The update stage, as described in Moës et al. (2002), is aimed at re-calculating the level sets following crack propagation. Extending to the $3 \mathrm{D}$ case, the corrections made by Duflot (2007) to the update equations reported in Moës et al. (2002), the level sets update can be described by means of the following equations (Colombo \& Massin, 2011):

$$
\begin{aligned}
& \frac{\partial \varphi_{n}(t)}{\partial t}+\nabla \varphi_{n}(t) \cdot \mathbf{V}_{\mathbf{N}}=0 \\
& \frac{\partial \varphi_{t}(t)}{\partial t}+\nabla \varphi_{t}(t) \cdot \mathbf{V}_{\mathbf{T}}=0
\end{aligned}
$$

with $\varphi_{n}$ and $\varphi_{t}$ the normal and tangential level sets, respectively; and $\mathbf{V}_{\mathbf{N}}$ and $\mathbf{V}_{\mathbf{T}}$ the propagation velocity at the crack front normal and tangential to the crack surface, respectively. Since Equations (1) are applied on the whole calculation domain but the velocity field is known at the crack front only, an extrapolation of the velocity is required. An example of such extrapolation can be found in Gravouil et al. (2002), which is based on the use of partial differential equations. This approach can be improved adopting an equivalent scheme based on the normal projection of each point $M$ of the domain on the crack front (Colombo \& Massin, 2011).

Given a point $P$ which is the projection of point $M$ on the crack front, the following velocity vectors are calculated:

$$
\begin{gathered}
\mathbf{V}_{\mathbf{N}}^{P}=\left(\mathbf{V}^{P} \cdot \mathbf{n}^{P}\right) \mathbf{n}^{P}=V_{\mathbf{N}}^{P} \mathbf{n}^{P} \\
\mathbf{V}_{\mathbf{T}}^{P}=\left(\mathbf{V}^{P} \cdot \mathbf{t}^{P}\right) \mathbf{t}^{P}=V_{\mathbf{T}}^{P} \mathbf{t}^{P}
\end{gathered}
$$


where $\mathbf{V}^{P}$ is the propagation velocity vector at point $P$ and $\left(\mathbf{t}^{P}, \mathbf{n}^{P}\right)$ are the vectors tangent and normal to the crack surface at the same point, respectively.

For the evolution of the tangential level set at all the points not included in the crack opening, a velocity equal to the one at the projected point on the crack front is considered. On the contrary, a linear extrapolation is used for the calculation of the velocity assigned to the normal level set if $\varphi_{t}(M)>0$, whereas for the other points where $\varphi_{t}(M) \leq 0$, a null velocity is adopted in order to preserve the existing crack surface (Gravouil et al., 2002). Thus:

$$
\begin{aligned}
& \left\{\begin{array}{l}
V_{\mathbf{N}}^{M}=0 \quad \text { if } \quad \varphi_{t}(M) \leq 0 \\
V_{\mathbf{N}}^{M}=V_{\mathbf{N}}^{P} \cdot \frac{\varphi_{t}(M)}{V_{\mathbf{T}}^{P} \Delta t_{\text {tot }}}
\end{array} \text { if } \quad \varphi_{\mathrm{t}}(M)>0\right. \\
& V_{\mathbf{T}}^{M}=V_{\mathbf{T}}^{P}
\end{aligned}
$$

where $\Delta t_{\text {tot }}$ represents the time or the number of fatigue cycles taken into account in the propagation step. For any point $M$, the velocity vectors of Equations (1) are finally calculated as follows:

$$
\begin{aligned}
& \text { if } \quad \varphi_{t}(M) \leq 0 \quad\left\{\begin{array}{c}
\mathbf{V}_{\mathbf{N}}^{M}=0 \\
\mathbf{V}_{\mathbf{T}}^{M}=V_{\mathbf{T}}^{P} \nabla \varphi_{t}(M)
\end{array}\right. \\
& \text { if } \quad \varphi_{t}(M)>0 \quad\left\{\begin{array}{l}
\mathbf{V}_{\mathbf{N}}^{M}=V_{\mathbf{N}}^{M} \mathbf{n}^{\mathbf{P}} \\
\mathbf{V}_{\mathbf{T}}^{M}=V_{\mathbf{T}}^{P} \mathbf{t}^{\mathbf{P}}
\end{array}\right.
\end{aligned}
$$

$\left(\varphi_{t}, \varphi_{n}\right)$ are the distances to the iso-zero tangential and normal level sets which are orthogonal to each other; therefore, $\left(\nabla \varphi_{t}, \nabla \varphi_{n}\right)$ define an orthonormal base at the nodes where $\left(\varphi_{t}, \varphi_{n}\right)$ are estimated. For all the points ahead of the crack characterised by $\varphi_{t}>0$, precise computations need to be conducted in order to locate the iso-zero surface of the normal level set and the new crack front. The level sets evolution is governed by the crack front evolution, with a propagation direction given in the $\left(\mathbf{t}^{P}, \mathbf{n}^{P}\right)$ local base at the crack front, which is not necessarily coincident with the level set gradient base $\left(\nabla \varphi_{t}, \nabla \varphi_{n}\right)$, as shown in the following part of this section. For all the other points lying in the crack opening, for which $\varphi_{t}<0$, less precise computations may be performed since the iso-zero of the normal level set is already fixed and independent of the crack front evolution: $\varphi_{t}$ is the only one to be modified and the direction of propagation for the $\varphi_{t}<0$ domain is then given in a simple way by the tangential level set gradient $\nabla \varphi_{t}$.

The calculation of the local base $\left(\mathbf{t}^{P}, \mathbf{n}^{P}\right)$ of Equations (4) is completely reviewed in relation to what is done in Gravouil et al. (2002), whose authors use the level set gradient interpolations. Yet, if the level set gradients are basically orthogonal to each other by construction at the nodes in the immediate vicinity of the crack, this is not the case for their interpolations, which causes errors in the extension stages (4). This point was already raised by Duflot (2007) who proposes the construction of a local base by favouring the normal level set and its gradient, with respect to the tangent one, for the construction of the base. Two reasons justify this choice: the accurate updating of the normal level set is vital when there is a change of direction in the crack propagation and the linear variation of the normal velocity in relation to the distance at the crack opening during the extension stage.

An improvement to what is done in Duflot (2007) is thus made, which allows obtaining a smooth velocity field all over the calculation domain. The crack front is represented piecewise linearly as in Fries and Baydoun (2011) by connecting the points where it intersects the 
elements faces. The local base can be calculated at these points by means of the aforementioned method (points $I$ and $J$ of Figure 1), whereas for any other position (point $P$ of Figure 1) on each crack front segment, it can be calculated from the ones at the two ends of the segment. For that, we use a rigid rotation which turns the local base at one segment end into the local base at the other end. Euler's theorem assures that this rotation is unique and is defined by its axis of rotation and its angle of rotation $\theta E$.

The position of $P$ on the segment $I J$, defined by $s=\|\overrightarrow{\mathrm{IP}}\| /\|\overrightarrow{\mathrm{IJ}}\|$, is used to determine the intermediate rotation from the local base at $I$ to the local base at $P$ in Figure 1, allowing to define the rotation angle $\theta_{E}^{P}=s \theta_{E}$ around the Euler axis previously defined for segment $I J$. Similarly, by means of a propagation law, the crack propagation velocity is calculated only at the points defining the crack segments. To ascertain the velocity at any point $P$ on a crack segment, the position $s$ of point $P$ on the crack segment is used once again, thereby enabling us to write (Figure 1):

$$
\left\|\mathbf{V}^{\mathrm{P}}\right\|=\left(\left\|\mathbf{V}^{\mathrm{J}}\right\|-\left\|\mathbf{V}^{\mathrm{I}}\right\|\right) \cdot s+\left\|\mathbf{V}^{\mathrm{I}}\right\|
$$

The propagation direction $\mathbf{V}^{\mathrm{P}}$ in the plane $\left(\mathbf{t}^{P}, \mathbf{n}^{P}\right)$ is thus obtained in a similar way by using linear interpolation between the angles formed by the vectors $\left(\mathbf{V}^{\mathrm{I}}, \mathbf{t}^{\mathrm{I}}\right)$ and $\left(\mathbf{V}^{\mathrm{J}}, \mathbf{t}^{\mathrm{J}}\right)$, or $\theta_{\mathrm{I}}$ and $\theta_{J}$, respectively. The velocity vector $\mathbf{V}^{\mathrm{P}}$ is therefore expressed as follows (Figure 1):

$$
\mathbf{V}^{\mathrm{P}}=\left\|\mathbf{V}^{\mathrm{P}}\right\|\left[\cos \left(\left(\theta_{J}-\theta_{\mathrm{I}}\right) s+\theta_{\mathrm{I}}\right) \mathbf{t}^{P}+\sin \left(\left(\theta_{J}-\theta_{\mathrm{I}}\right) s+\theta_{\mathrm{I}}\right) \mathbf{n}^{P}\right]
$$

Being $\mathbf{V}^{\mathrm{P}}$ and the local base $\left(\mathbf{t}^{P}, \mathbf{n}^{P}\right)$ known, Equations (1) can be completely solved.

Despite these corrections, it has been shown that numerical instabilities appear when the crack tips drastically change direction (Colombo \& Massin, 2011). Moreover, in such a case, if an explicit integration scheme is used, to solve the Hamilton-Jacobi Equations (1), the time step must be drastically reduced to satisfy the CFL (Courant-Friedrichs-Lewy) condition, which is detrimental to the calculation performance. This problem may be avoided if an implicit integration scheme is used but at the expense of an increased complexity in the calculation code programming. Recently, a solution with one sole resolution iteration, based on the use of the "fast marching method", was put forward in Sukumar, Chopp, Béchet, and Moës (2008), Shi, Chopp, Lua, Sukumar, and Belytschko (2010). However, as indicated in

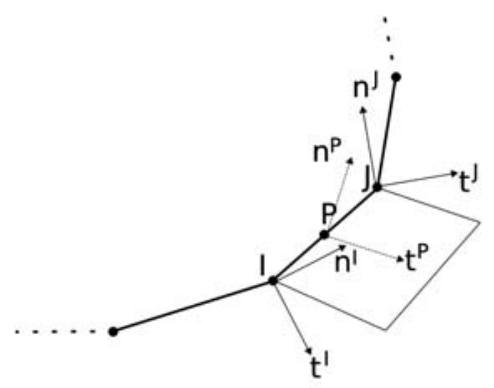

(a)

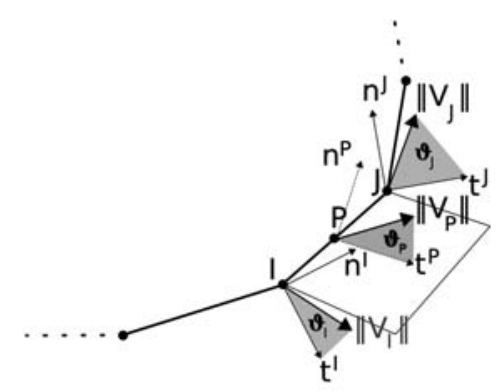

(b)

Figure 1. Local bases at the crack opening. (a) Use of local bases at $I$ and $J$, points of intersection of the crack front with the X-FEM finite elements, to determine the local base at any point $P$ of the crack front inside the element by using Euler's rotation, which means that the local base at $I$ can be turned into the local base at $J$. (b) The propagation velocity is only known at points $I$ and $J$. The velocity at point $P$ is calculated by using the velocity at these points. 
Sukumar et al. (2008), the calculated solution is susceptible to errors near to the boundaries of the solid. Several ingredients are therefore required to solve the problem of instability.

An alternative solution is put forward in Colombo and Massin (2011) and Fries and Baydoun (2011) which is based on an explicit polynomial equation, and which does not require an integration scheme or an iterative resolution. The method proposed here is simpler than the one of Fries and Baydoun (2011) which relies on the use of three level sets instead of two combined with a physical geometrical extension of the crack surface, as in Geniaut and Massin, which we do not need here. It means that good performance and resolution stability can go hand in hand, while having only a slight computing impact on the code. The evolution of the level sets is thus calculated in the following way, which represents the discontinuous form of (1):

$$
\begin{aligned}
\Delta \varphi_{n} & =-\left(\nabla \varphi_{n}(t) \cdot \mathbf{V}_{\mathbf{N}}^{M}\right) \Delta t_{\text {tot }} \\
\Delta \varphi_{t} & =-\left(\nabla \varphi_{t}(t) \cdot \mathbf{V}_{\mathbf{T}}^{M}\right) \Delta t_{\mathrm{tot}}
\end{aligned}
$$

With respect to (1), formulation (7) presents the advantage of not having to verify the CFL condition in the event that explicit resolution algorithms, as the Runge-Kutta family, are used. This is the most restrictive point to be solved since in the normal level set propagation equation (first equation of (1)) the velocity $\mathbf{V}_{\mathbf{N}}$ increases linearly with the distance to the crack front (see (3)), causing the critical time step to decrease proportionally, thereby reducing the performance of the algorithm and preventing the important crack deviation angles from being treated numerically. The methodology put forward here enables us to treat crack propagation at almost $90^{\circ}$, which is impossible to model as of (1) (Colombo \& Massin, 2011).

\subsection{Numerical resolution of the re-initialisation and re-orthogonalisation stages}

After the update stage of the level sets, their property of distance to the crack front is not necessarily maintained and neither is the orthogonal property between the two level sets. It is therefore necessary to proceed to the following corrective stages (Gravouil et al., 2002):

- The normal level set is re-initialised so that a distance function can be re-established $\left(\left\|\nabla \varphi_{n}\right\|=1\right)$ :

$$
\frac{\partial \varphi_{n}}{\partial \tau}=-\operatorname{sign}\left(\varphi_{n}\right)\left(\left\|\nabla \varphi_{n}\right\|-1\right)
$$

- The tangent level set is re-orthogonalised in relation to the normal level set $\left(\nabla \varphi_{n} . \nabla \varphi_{t}=0\right)$ :

$$
\frac{\partial \varphi_{t}}{\partial \tau}=-\operatorname{sign}\left(\varphi_{n}\right) \frac{\nabla \varphi_{n}}{\left\|\nabla \varphi_{n}\right\|} . \nabla \varphi_{t}
$$

- The tangent level set is re-initialised so that a distance function can be re-established $\left(\left\|\nabla \varphi_{t}\right\|=1\right)$ :

$$
\frac{\partial \varphi_{t}}{\partial \tau}=-\operatorname{sign}\left(\varphi_{t}\right)\left(\left\|\nabla \varphi_{t}\right\|-1\right)
$$


Equations (8)-(10) are solved until their fixed points are set, with $\tau$ as a virtual time, as opposed to Equations (1). It can also be remarked that they all have the same form which is expressed in fine as:

$$
\frac{\partial \varphi}{\partial \tau}+\mathbf{u} . \nabla \varphi=c
$$

As soon as the iso-surfaces of the level sets are formed, having ascertained the velocity at the crack front, the order in which the above stages are executed becomes important. Indeed, in case of curved cracks, it is not possible to form level sets which verify Equations (8)-(10) at the same time, at each point of the domain (but the Equations (8)-(10) will be verified at the crack front). In order to easily obtain the distance at the crack front, we end the process by re-initialising each family of level sets.

In order to efficiently integrate the equations of the form (11), the simplex method of Barth and Sethian (1998) has been adopted in Gravouil et al. (2002) and Geniaut and Massin. The advantage of this method, of which a detailed description can be found in Geniaut and Massin, is that it can be directly applied to $2 \mathrm{D}$ triangular and 3D tetrahedral meshes. Furthermore, meshes of industrial structures, which are often non-structured, may easily be split into triangles or tetrahedral elements.

Despite this advantage, based on the experience of the authors, this is not necessarily the most stable of the methods. Methods of finite difference type have proven to be highly interesting: initially used in 2D (Prabel, Combescure, Gravouil, \& Marie, 2009), in the framework of dynamic crack propagation, their use has recently been extended to 3D (Sukumar et al., 2008) on simple examples in association with the "fast marching method".

Based on the work of Prabel et al. (2009) in 2D, a finite difference scheme of the first order based on a regular grid is established. This solution requires a projection of the level sets between the grid and the finite element mesh, where the mechanical calculations are carried out. Two obstacles must overcome in relation to this kind of algorithm:

- the side effects at the domain boundaries, which introduce stray reflections and favours instability,

- the performance, if the calculation domain is not localised (Colombo \& Massin, 2011). In order to improve it, a restriction on the projection domain is proposed, as well as a localisation around the crack front of the domain where Equations (8)-(10) are solved. The localisation also allows improving the stability of the algorithm. A similar solution put forward by Shi et al. (2010) is improved herein.

Equation (11) is a transport equation, propagating the information in the direction of $\mathbf{u}$ from the iso-zero surface to the whole domain. Only information obtained in the windward direction is used to find the solution. Looking at the form of Equation (11), the points on the boundaries of the domain, for which the integration scheme cannot be used in its current form, can be easily identified. A 2D illustration is provided in Figure 2. For the points in black, the points below are missing, whereas for the points in grey the information about the points situated above would be required.

In order to solve this problem, the "phantom point technique" is used in fluid dynamics (Randal, 2002), which is based on an extrapolation of information using the nearby points and physical fields such as pressure or velocity. In our case, the extrapolation process has to be carried out on a less meaningful physical quantity over which it is more difficult, if not 


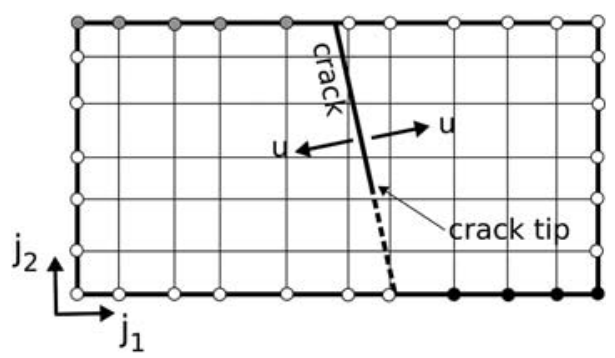

Figure 2. Example of a 2D propagation. Auxiliary grid showing problematic points (black and grey points) at boundary.

impossible, to exercise control. Therefore, we avoid employing this method and we suggest the direct introduction of the missing information at the problematic points on the boundary by directly calculating level sets values, that is for the re-initialisation stages; the distance to the iso-zero surface of the level set being re-initialised; and for the re-orthogonalisation stage, the value of the tangent level set at the orthogonally projected point on the normal level set iso-zero (Colombo \& Massin, 2011).

\section{Level sets geometrical calculation as an alternative to level sets propagation}

By combining the ideas at the basis of the velocity extension process (Section 2.1), of the formulation of Equations (7) and of the domain localisation for the re-initialisation and reorthogonalisation stages (Section 2.2), a fully geometrical approach for level set re-calculation at each propagation step can be formulated (Colombo, 2012). This enables robustness and fast calculation, because all the stages presented above are condensed in one stage for which only explicit geometrical equations are used. In fact, now being clear from the discussion and the equations presented above that the problem is always a $2 \mathrm{D}$ problem in the $\left(\mathbf{t}^{P}, \mathbf{n}^{P}\right)$ plane of point $\mathrm{P}$, projection of a generic point $\mathrm{M}$ of the calculation domain, the two level sets can be re-calculated directly by means of the following equations (see Figure 3):

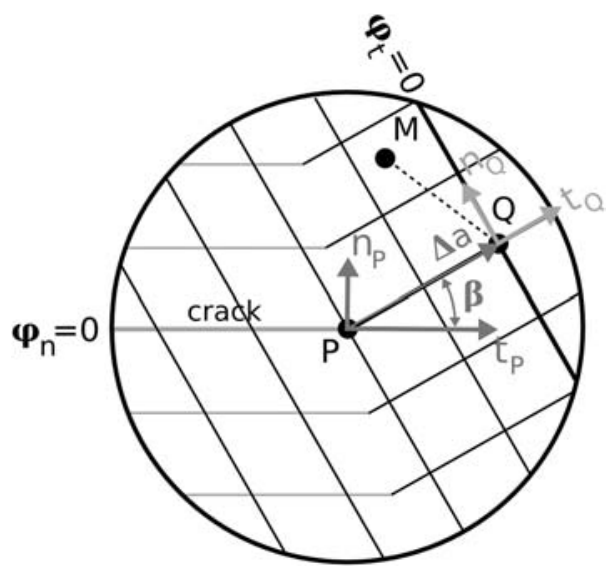

Figure 3. Distance to the crack front. Local base at the new position $Q$ of the crack front and isosurfaces in the $\left(\mathbf{t}^{p}, \mathbf{n}^{p}\right)$ plane after application of (7)-(10). 


$$
\begin{aligned}
\varphi_{n}(M) & =\overrightarrow{M Q} \cdot \mathbf{n}^{Q} \\
\varphi_{t}(M) & =\overrightarrow{M Q} \cdot \mathbf{t}^{Q}
\end{aligned}
$$

Point $\mathrm{P}$ is the projection of point $\mathrm{M}$ on the actual crack front and $\mathrm{Q}$ is its new position after the propagation. Its position can be found by adding to the coordinates of point $\mathrm{P}$ the propagation vector $\Delta \vec{a}$ at point $\mathrm{P}$, which is known with both the crack advancement $\Delta \mathrm{a}$ and the crack propagation angle $\beta$ obtained from the propagation law. Similarly, the local base $\left(\mathbf{t}^{Q}, \mathbf{n}^{Q}\right)$ can be calculated by a rotation of the local base $\left(\mathbf{t}^{P}, \mathbf{n}^{P}\right)$ around the axis $\mathbf{t}^{P} \wedge \mathbf{n}^{P}$. In order to preserve the normal level set iso-surfaces in the vicinity of the existing crack surface, Equations (12) are applied only at the points for which the tangential level set is positive.

In case a point $\mathrm{M}$ has no normal projection on the actual crack front (Figure 4), point $\mathrm{P}$ is taken coincident with one end of the crack front as in Colombo and Massin (2011) or Geniaut and Massin and both level sets are always evaluated by means of Equations (12) by substituting $\mathbf{t}^{P^{*}}$ to $\mathbf{t}^{P}$ (Figure 4 ) so as to obtain the local base $\left(\mathbf{t}^{Q}, \mathbf{n}^{Q}\right)$. $\mathbf{t}^{P^{*}}$ is parallel to $P M^{*}$, where $M^{*}$ is the projection of point $M$ on the plane orthogonal to $\mathbf{n}^{P}$ at point $P$. It is oriented in such a way that the scalar product $\mathbf{t}^{P} \bullet \mathbf{t}^{P^{*}}$ remains positive at point $\mathrm{P}$, except when $\mathbf{t}^{P} \bullet \mathbf{t}^{P^{*}}=0$ in which case the orientation of $\mathbf{t}^{P^{*}}$ is given by $\mathbf{P} \mathbf{M}^{*}$.

Equations (12) allow the elimination not only of the velocity extension step and the update phases, but also of the re-initialisation and re-orthogonalisation stages, which results in a huge decrease of the computational time and increases the stability of the algorithm. This is further enhanced by the fact that neither the grid projection nor the domain localisation is necessary anymore. Finally, it must be remarked that Equations (12) can successfully manage fronts splitting and merging during the propagation (Colombo, 2012).

\section{Example of out-of-plane 3D crack propagation}

A three-point flexural precracked sample is modelled as shown in Figure 5 (Lazarus, Buchholz, Fulland, \& Wiebesiek, 2008). The crack is initially inclined with respect to the loading direction, which means that a considerable twisting of the crack surface is observed thereby enabling the numerical choices we have put in place to be validated in a relevant way. The crack displays a mixed-mode charge I + II + III with a crack front which twists to propagate in mode I. This evolution has been modelled in Citarella and Buchholz (2008): a qualitative evolution of the solution may only be given as the information on the law of

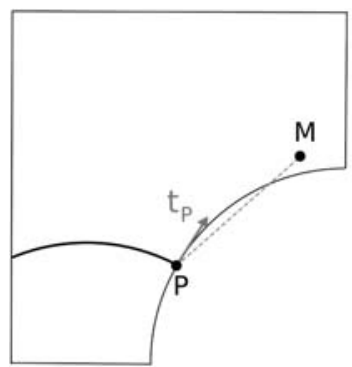

(a)

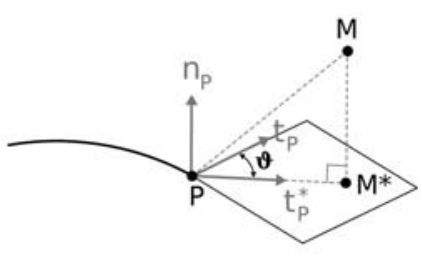

(b)

Figure 4. Distance to the crack front. For points that have no projection on the discretised iso-zero normal level set. (a) Point $M$ has no projection on the crack front. (b) Local base rotation adopted to correctly calculate the distance of point $M$ to the crack front. 


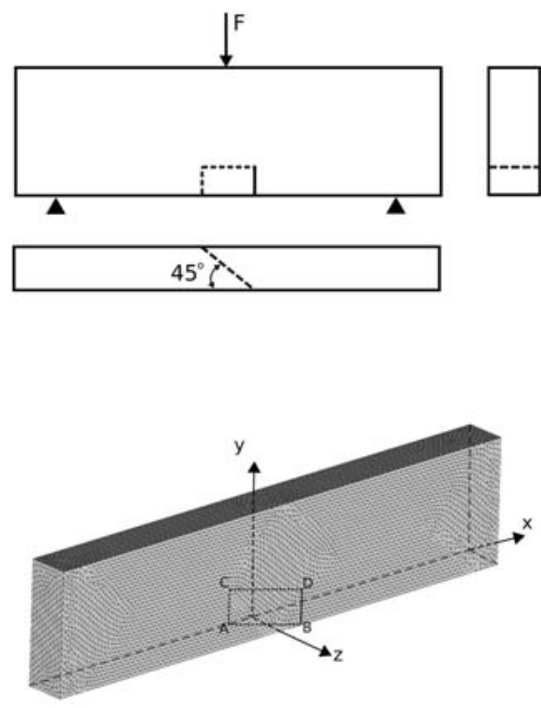

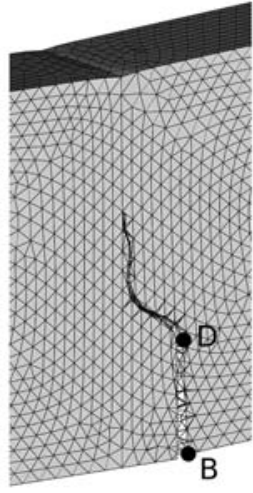

(a)

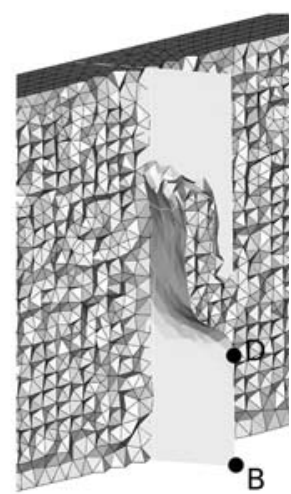

(b)

Figure 5. Crack formation path for a crack inclined at $45^{\circ}$ in relation to the axis of a three points flexural sample. On image (a), the sample is distorted with an amplification factor of five on the displacements. On image (b) the iso-zero surface of the normal level set can be seen superimposed on the plane of the initial crack, which means that the twisting of the crack can be seen so that stress in mode I can be found.

propagation used in Citarella and Buchholz (2008) is not provided. In that case, in order to sufficiently refine the mesh around the crack front for fracture mechanics calculations, the automatic re-meshing procedure of Code_Aster which makes use of Homard software is employed. This refinement does not concern the level sets calculation grid because that would not make any improvement, since the interpolation required to go from one grid to the other would not add any information.

The direction of propagation used in Citarella and Buchholz (2008) is the one given by the minimum local energy density of Sih (1974). This direction is not influenced by the value of the $\mathrm{K}_{\mathrm{III}}$ stress intensity factor. As a matter of fact, mode III activation is linked to the rotation of the crack front with respect to $\mathbf{t}_{\mathbf{P}}$, which cannot be represented by a regular continuous crack front. Experimental results of Lazarus et al. (2008) show a discontinuous factory roof profile of the crack front characterising a mode III presence. However, the general evolution of the crack profile can be obtained with a local criteria of maximum normal stress (Erdogan \& Sih, 1963), similar to the one of Sih (1974), which just takes into account mode I and II.

The advance of crack tips for each calculation step is shown in Figure 6 both for Code_Aster and for Citarella and Buchholz (2008). Similar trends are observed with crack fronts which are a little more curved in our case (compare top left of Figure 6 to bottom left of Figure 6), which could be caused by fatigue material parameters which are slightly different but not shown in Citarella and Buchholz (2008).

In order to compare the results, the numerical solution has been calculated twice, once by means of the level set propagation technique presented in Section 2 and once by means of the geometrical approach presented in Section 3. Concerning the level set propagation technique, an auxiliary regular grid has been used for the calculations and the updated level sets have been projected on the finite element mesh. In particular, the update phase has 

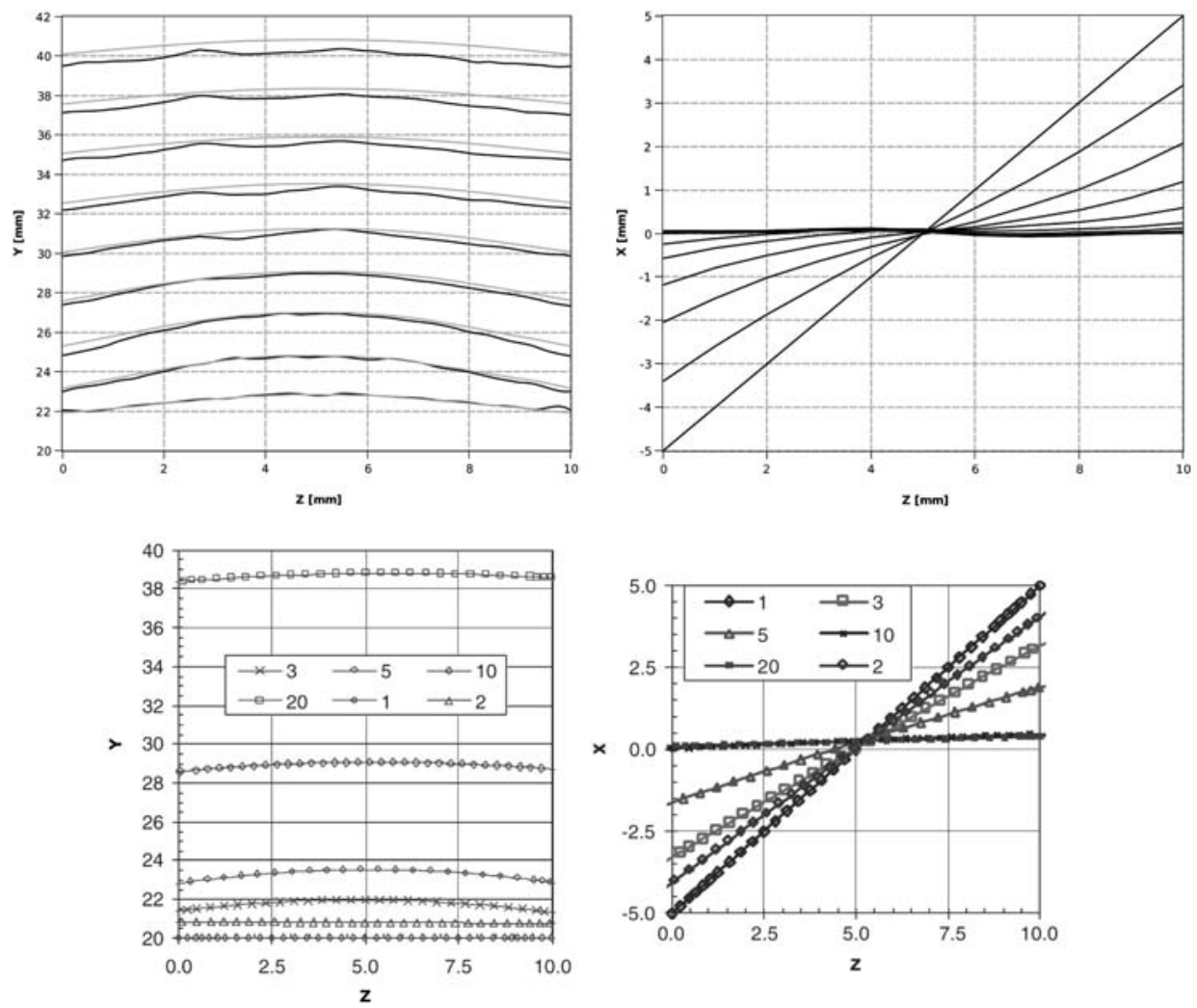

Figure 6. Crack tips obtained for each propagation step. The results (vertical section on the left $Y-Z$ and horizontal section on the right $X-Z$ ) obtained with Code_Aster are given in the upper section (in the $Y-Z$ plot on the left, black lines refers to the level set update methodology, grey lines to the recalculation methodology, in the $X-Z$ plot on the right the results correspond to the re-calculation) and those obtained by Citarella and Buchholz (2008) are displayed in the lower section.

been carried out by means of the explicit Equations (7); and the re-initialisation and reorthogonalisation phases have been carried out by solving the set of the three PDEs (partial differential equation) (8)-(10) by means of a first-order upwind finite difference scheme, as detailed in Colombo and Massin (2011).

The solutions obtained with these two numerical techniques are similar. They are shown in Figure 6. The left part of Figure 6 exhibits crack profiles in vertical sections parallel to the $Y-Z$ plane, while the right part exhibits crack profiles in horizontal sections parallel to the $X_{-}$ $Z$ plane; while propagating, the crack initially at $45^{\circ}$ re-assumes a $90^{\circ}$ orientation. The solutions of Figure 6 in plane $Y-Z$ show that the geometrical approach technique produces smooth crack fronts, whereas the level set update technique produces minor oscillations, which get smaller and smaller as the auxiliary grid is refined or as the crack advancement used in the simulation step is reduced. As already stated in Section 2.1, small errors in the calculation of crack advancement and velocity at the crack front are amplified far from the front due to the linear interpolation of the normal velocity (Equation (3)). Small oscillations appear in the recalculated values of the level sets between two adjacent points of the grid. Such errors cumulate with the propagation steps since the level set is updated for a given step from the values of the previous step. This is not the case for the geometrical technique, where the level sets are re-calculated but not updated from a previous propagation step. Moreover, the finite dif- 
ference scheme used in the level set update technique requires a finer mesh as the value of the level set gradients increases.

If performances of both the techniques are compared, the computational time required with the geometrical technique is $70-80 \%$ lower or $3-5$ times faster than the one required with the update based on Hamilton-Jacobi equations (see Colombo \& Massin, 2011).

\section{Conclusions}

An overview of different techniques for crack propagation simulation has been presented, with a crack surface model based on level sets and their update after propagation steps. In particular, the discussion focused on solutions which can be adopted to solve the drawbacks and the stability problems experienced in 3D high kink angle crack propagation simulations. The level sets geometrical calculation technique presented in Section 3 is the most promising both in terms of accuracy, robustness and performance. Moreover, it enables the propagation of cracks in sections with holes, as shown in Colombo (2012), making it possible to automatically manage the division of the crack front in several fronts and the merging of several fronts into a single one. This feature is still the object of ongoing research efforts in order to simulate flawlessly 3D high kink angles crack fronts dividing and merging. For all these reasons, this methodology will be the one promoted for crack propagation in Code_Aster in the very next years.

\section{Note}

1. The Homard software is a free software, which is part of the software set available with Code_Aster. Please see http://www.code-aster.org/outils/homard for further information about this tool.

\section{References}

Adalsteinsson, D., \& Sethian, J.A. (1995). A fast level set method for propagating interface. Journal of Computational Physics, 118, 269-277.

Barth, T.J., \& Sethian, J.A. (1998). Numerical schemes for the Hamilton-Jacobi and level set equations on triangulated domains. Journal of Computational Physics, 145, 1-40.

Belytschko, T., \& Black, T. (1999). Elastic crack growth in finite elements with minimal remeshing. International Journal for Numerical Methods in Engineering, 45, 601-620.

Citarella, R., \& Buchholz, F.G. (2008). Comparison of crack growth simulation by DBEM and FEM for SEN-specimens undergoing torsion or bending loading. Engineering Fracture Mechanics, 75, 489-509.

Colombo, D. (2012). An implicit geometrical approach to level sets update for 3D non planar X-FEM crack propagation. Computer Methods in Applied Mechanics and Engineering, 237-240, 39-50.

Colombo, D., \& Massin, P. (2011). Fast and robust level set update for 3D non-planar XFEM crack propagation modelling. Computer Methods in Applied Mechanics and Engineering, 200, 2160-2180.

Duflot, M. (2007). A study of the representation of cracks with level sets. International Journal for Numerical Methods in Engineering, 70, 1261-1302.

Erdogan, F., \& Sih, G. (1963). On the crack extension in plates under plane loading and transverse shear. Journal of Basic, Engineering, 85, 519-527.

Fries, T.P. \& Baydoun, M. (2011). Crack propagation with the extended finite element method and a hybrid explicit-implicit crack description. International Journal for Numerical Methods in Engineering, DOI:10.1002/nme.3299.

Geniaut, S., \& Massin, P. Extended finite element method. Reference documentation [R7.02.12] of Code-Aster. Available on the project website: www.code-aster.org

Gravouil, A., Moës, N., \& Belytschko, T. (2002). Non planar 3D crack growth by the extended finite element and level sets - Part II: Level set update. International Journal for Numerical Methods in Engineering, 53, 2569-2586.

Lazarus, V., Buchholz, F.G., Fulland, M., \& Wiebesiek, J. (2008). Comparison of predictions by mode II or mode II criteria on crack front twisting in three of four bending experiments. International Journal of Fracture, 153, 141-151. 
Moës, N., Dolbow, J., \& Belytschko, T. (1999). A finite element method for crack growth without remeshing. International Journal for Numerical Methods in Engineering, 46, 135-150.

Moës, N., Gravouil, A., \& Belytschko, T. (2002). Non planar 3D crack growth by the extended finite element and level sets - Part I: Mechanical model. International Journal for Numerical Methods in Engineering, 53, 2459-2568.

Peng, D., Merriman, B., Osher, S., Zhao, H., \& Kang, M. (1999). A PDE-based fast local level set method. Journal of Computational Physics, 155, 410-438.

Prabel, B., Combescure, A., Gravouil, A., \& Marie, S. (2009). Level set X-FEM non matching meshes: Application to dynamic crack propagation in elastic-plastic media. International Journal for Numerical Methods in Engineering, 78(12), 1407-1435.

Randal LeVeque, J. (2002). Finite volume methods for hyperbolic problems. Cambridge, UK: Cambridge University Press.

Shi, J., Chopp, D., Lua, J., Sukumar, N., \& Belytschko, T. (2010). Abaqus implementation of extended finite element method using a levet representation of three-dimensional fatigue crack growth and life predictions. Engineering Fracture Mechanics, 77, 2840-2863.

Sih, G. (1974). Strain energy density factor applied to mixed mode crack problems. International Journal of Fracture, 10, 305-321.

Sukumar, N., Chopp, D.L., Béchet, E., \& Moës, N. (2008). Three-dimensional non-planar crack growth by a coupled extended finite element and fast marching method. International Journal for Numerical Methods in Engineering, 76, 727-748.

Ventura, G., Budyn, E., \& Belytschko, T. (2003). Vector level sets for description of propagating cracks in finite element. International Journal for Numerical Methods in Engineering, 58, 1571-1592. 